\title{
МОДЕЛИРОВАНИЕ АДГЕЗИОГЕНЕЗА БРЮШНОЙ ПОЛОСТИ И ЕГО ПРОФИЛАКТИКА В ЭКСПЕРИМЕНТЕ
}

\begin{abstract}
MODELING OF ABDOMINAL
ADHESIOGENESIS AND ITS PREVENTION
IN AN EXPERIMENT
M. Magomedov

M. Magomedov

M. Magomedov

Summary. Meshes implanted intraperitoneally are known to cause adhesions, potentially leading to complications such as chronic pain, intestinal fistulas, infection, and adhesions. To model intestinal adhesions, a polyprolene mesh was used and its role in the development of adhesions was evaluated. This study introduces a model for investigating intestinal adhesions to the mesh and assesses whether visceral peritoneal damage is causal to the development of adhesions. Experimental modeling of postoperative intraabdominal adhesions using a polyproline mesh. Experiments were performed on 24 healthy dogs weighing $10-15 \mathrm{~kg}$. At the first stage, the adhesive process was modeled using a $4 \times 4 \mathrm{~cm}$ polyproline mesh fixed on the visceral surface of the cecum and deserization of the parietal peritoneum of the right mesogastric region. At the second stage, 14 days later, a relaparatomy and revision of the abdominal organs were performed with an assessment of the adhesive process. With the development of the adhesive process, the adhesions were dissected and removed from the visceral surface of the mesh. Further, randomization was performed with the division of experimental dogs into 4 groups using an amneotic membrane, 5-fluorouracil and mesogel. The fourth control group with intraperitoneal administration of $5 \%$ glucose. In an experiment on the model, it was found that the main condition for the formation of adhesion is damage to the mesothelium of the serous cover of the intestine by a mesh. The results of caecum adhesion to the mesh according to the result of Lauder $\mathrm{C}-92 \%$, and Hoffman $\mathrm{N}-90 \%$. Histological examination showed adequate ingrowth of the mesh into the wall in all animals. The most promising results were obtained with the use of an amniotic membrane implant and 5 - fluorouracil, while the worst results were obtained with the use of mesogel. There were also statistically significant differences between the groups after histopathological examination.
\end{abstract}

Keywords: intraperitoneal adhesions, polypropylene mesh, adhesion formation, experimental study, caecum.

\author{
Магомедов Мухума Магомедович \\ Дагестанский Государственный Медицинский \\ Университет \\ muxuma@mail.ru \\ Магомедов Магомед Арсеневич \\ Дагестанский Государственный Медицинский \\ Университет \\ magomedovma@rushydro.ru \\ Магомедов Магомед Абдулхаликович \\ Дагестанский Государственный Медицинский \\ Университет \\ kafedra.khirurgii@mail.ru
}

Аннотация. Сетки, имплантированные внутрибрюшинно, как известно, вызывают спайки, потенциально приводящие к осложнениям, таким как хроническая боль, кишечные свищи, инфицирование, спайки. Для моделирования спаек кишечника использовали полипроленовая сетка и оценивали, ее роль развития спаек. Это исследование вводит модель для исследования спаек кишечника к сетке и оценивает, является повреждения висцеральной брюшины причинным развития спаек. Моделирование в эксперименте послеоперационных интраабдоминальных спаек с применением полипроленовой сетки. Эксперименты проводили на 24 здоровых собаках с весом 10-15 кг. На первом этапе моделировали спаечный процесс с применением полипроленовой сеткой фиксированной на висцеральной поверхности слепой кишки размером 4 × 4 см и десерозированием париетальной брюшины правой мезогастральной области. На втором этапе спустя 14 суток выполняли релапаратомию и ревизию органов брюшной полости с оценкой спаечного процесса. При развитии спаечного процесса проводили рассечение спаек и удаление из висцеральной поверхности сетки. В дальнейшем проводили рандомизация с разделением экспериментальных собак на 4 группы с применением амнеотической мембраны, 5-фторурацила и мезогеля. Четвертая группа контроля с интраперитониальным введением 5\% глюкозы. В эксперименте на модели, установлено, что основным условием образования адгезии является повреждение мезотелия серозного покрова кишки сеткой. Результаты адгезии слепой кишки в сетку по результату Lauder C - 92\%, a Hoffman $\mathrm{N}-90 \%$. Гистологическое исследование показало адекватное врастание сетки в стенку у всех животных. Наиболее обнадеживающие результаты получены с применением имплантата — амниотической мембраны и 5-фторурацила, худшие результаты с применением мезогеля. Также наблюдалось статистически значимые различия между группами после гистопатологического исследования.

Ключевые слова: внутрибрюшинные спайки, полипропиленовая сетка, формирование адгезии, экспериментальное исследование, слепая кишка. 


\section{Ввемение}

$\Pi$ оследствиями образования адгезии могут быть хронические боли в животе [1, с. 108; 5, с. 328], женское бесплодие [5, с. 328; 2, с. 34; 8, с. 132; 9 , c. 544], кишечная непроходимость [5, с. 329; 6, с. 378; 7, c. $346 ; 10$, с. 1568]. Кроме того, спайки могут серьезно осложнить повторные операции $[9,10]$ и в целом увеличить риск повторной операции. В зависимости от хирургической техники и типа сетки, частота образования адгезии около $30 \%$ сообщается [12, с. 78; 21, с. 1267]. Послеоперационные спайки формируются после травмы брюшной полости и является результатом биохимической и клеточной реакции $[21$, с. 1268,14, с. $878 ; 16$, c. 67]. Хотя спайки оказывают не благополучное воздействие, они являются ведущей причиной развития острой спаечной кишечной непроходимости и могут быть источником значительной заболеваемости, в некоторых случаях приводящей к летальному исходу [11, c. $377 ; 17$, c. 275].

Модель с применением полипроленового имплантата обеспечивает высокую воспроизводимость адгезионного образования и полезна для тестирования и сравнения различных устройств предотвращения адгезии [19, с. 5; 15]. В настоящем исследовании представлен новый вариант модели с имплантацией сетки в правую подвздошную область в проекции слепой кишки для моделирования спаечной болезни. Статистический анализ. Все значения выражались, как средняя +SE. Категориальные параметры в каждой группе сравнивались с помощью X2 и инепреревистые параметры сравнивались с использованием независимого теста. Все анализы проводили с использованием SPSS12.0 и результаты считались статистически значимыми $(p<0,05)$. Целю исследования является моделирование в эксперименте послеоперационных интраабдоминальных спаек с применением полипроленовой сетки.

\section{Материалы и методы}

Экспериментальное исследование выполняли с соблюдением принципов, изложенных в Конвекции по защите животных, использованных для экспериментальных исследований (Страсбург, Франция, 1986). Все экспериментальные животные самцы весом от10 до 14 кг. В условиях стерильности операционного блока в институте Экологической медицины Дагестанского государственного медицинского университета были проведены экспериментальные моделирования спаечного процесса в брюшной полости у 24 собак с применением полипроленовой сетки. Амнеотическая мембрана для имплантации готовили по методике Дубовикова А.С. [4, с. $214 ; 3$, с. 69].
Для достижения общей анестезии вводили зависимую от веса дозу 80 мг/кг кетамина и 5 мг/кг ксилазина после проведенной промедикации атропина - 0,1\% $(0,8)$ и дроперидола - 2,5 мл. Операции на экспериментальных собаках проводила в два этапа. Затем переднюю стенку брили и обрабатывали спиртом и раствором йода. Доступ к брюшной полости был получен с помощью 5-6 см срединной лапаротомии. Прямоугольные участки размером $5 \times 5$ см из непокрытой полипропиленовой сетки были прошиты швом 4/0 Prolene ${ }^{\circledast}$ на десерозированный висцеральной поверхности слепой кишки у 24 животных. Продолжительность оперативного вмешательства не превышало 25-30 мин. По завершении рана послойно заживали операции у всех оперированных животных.

Животные находились под наблюдением до полного пробуждения после операции вводили кетарол - 1 мл в/м, снижение ожидаемой боли в ближайшем послеоперационном периоде. На втором этапе спустя 14 суток выполняли повторную операцию с ревизией органов брюшной полости с изучением морфологические изменения в области предыдущего оперативного вмешательства. При развитии спаечного процесса проводили адгезиолизис в области повреждения и анатомических областях брюшной полости. Из области слепой кишки в проекции расположения эндопротеза подвергали предварительной гидравлической препаровке с введением 0,5\% новокаина и последующим удалением полипроленовой сетки. После удаление сетки появилось капиллярное кровотечение. После обработки тампоном перекисью водорода кровотечение остановлено. Слепой стеной кишки было 4 × 5 десерозированный участок. В дальнейшем проведено рандомизация вмешательства. Через 14 суток после операции оценивали макроскопически по шкале Nair [14, с. 70].

Первая группа: слепая кишка выведена в рану вместо десерозированного участка размером 4 × 4 см размещена амниотическая мембрана и проведена перетонизация участка методом ушивания этиконовой нитью ( $\mathrm{n}=$ 7). Вторая группа: после удаления сетки имелось десерозированный участок 4×4,2 см. Перед послойным ушиванием послеоперационной раны внутрибрюшно вводили 10 мл - 5-фторурацила на 0,9\% физиологическом растворе при температуре 120 а после операции - 1 мл сулодексида $(n=7)$. Третья группа: перед послойным ушиванием послеоперационной раны внутрибрюшно вводили 15 мл/кт раствора мезогель $(n=7)$. Четвертая группа: перед послойным ушиванием послеоперационной раны интраперитониально вводили 10 мл/кт раствора 5\% глюкозы $(n=7)$.

Спустя через две недели экспериментальные животные были оперировали для изучения оценки морфоло- 
гического процесса в брюшной полости. При вскрытии брюшной полости были использованы визуально-описательного метода. При лапаротомии были сделаны фотографии и пораженных участков всех оперированных животных для документирования.

В первой группе экспериментальных животных $\mathrm{n}=$ 7 развития послеоперационного процесса в области моделированного участка было различными по выраженности и распространенности процесса и характеру морфологических изменений брюшины. У 6 экспериментальных животных были представлен сращения состоящими из слепой кишки и сальника, к месту расположения имплантата. Макроскопическая оценка степени адгезии касс 1-2 по Nair. У 1-х животного было изолированное сражение сальника к операционному рубцу.

Во второй группе экспериментальных животных $\mathrm{n}=7$ развитие спаечного процесса по Nair по макроскопической структуре было у 5 собак 2 класс, у 2 собак класс 3. Сражения слепой кишки и сальника к месту расположения сетки были основном плоскостные спайки легко поддавались разделению тупым путем. Степень ремезотелизации поврежденных участков не превышало $48 \%$.

В третьей группе экспериментальных животных $\mathrm{n}=$ 7 в области моделирования спаечный процесс развился у всех животных. Патологический процесс был представлен сражениями, состоящими из тонкой кишки и сальника, плотный с трудом отделяемое сражения к месту расположения эндопротеза. Степень зрелости спаек варьировала от аваскулярных плоских $\mathrm{n}=2$, сражения слепой кишки с сальником $\mathrm{n}=3$. У 5 животных спаечный процесс распространялся на соседние тонкую и толстую кишку. В области малого таза абсцесс ограниченный абсцесс малого таза. Макроскопическая картина по степени адгезии по Nair 3-4 класса.

У четвертой группы $\mathrm{n}=7$ спаечный процесс в области моделирования развился у всех животных. У 2-х животных развился сражения в виде формирования конгломератов, состоящий из слепой кишки, толстой кишки и сальника. Место расположения сетки в области слепой кишки плотные сращения с толстой кишки с трудом отделяемое. Слепая кишка местами деформирована спаечной кишечной непроходимостью. Макроскопическая картина степени адгезии у всех животных было 3 и преимущественно 4 класса.

Использование для профилактики спаечного процесса мезогель и глюкоза 5\%, хотя имело приблизительно одинаковый эффект в общем снижение случаев спайкообразование, но принципиально не отличалось по характеру, распространенности и выраженности морфологических изменений брюшины $(p<0<001)$. Не- обходимо отметит инфицирование и абсцесса брюшной полости с применением мезогеля у 3-х животных. Более обнадеживающие результаты получены с применением амнеотической мембраны, выявлено меньшая толщина фиброзного рубца в зоне дефекта стенки кишки $(p<0,005)$ и меньше выраженное формирование фибринозных спаек в зоне повреждения.

Использование 5-фторурацила с сулодексидом было отмечено одинаковую частоту снижения случаев адгезиолизиса в сравнении 3 и 4 группой $(p<0,05)$. У собаки 3-4 группы развивали достоверно $(\mathrm{p}=0,0002)$ больше спаек слепой кишки к месту, где ранее была имплантирована сетка, чем у животных 1-2 группы: средний балл Lauder C[14] 4,6 \pm 1,3 (=92\%) и средний суммарный балл Hoffmann N [12] $9 \pm 2,8$ (=90\%) против $1,6 \pm 1,5$ (= 35\%) и 2,4 $\pm 3,2$ (= 24\%) 1-2 группы. Фиброза развилась 1-2 группы $(0,002)$ и воспаления $(p=0,003)$ и 3-и группы ( $p=0,003$ и 4-й $p=0,002)$ соответственно. При гистологическом исследовании значительное различие было отмечено в сравнениях между группами с точки зрения неоваскуляризации, плотности фибробластов, отложения коллагена и воспалительных клеток (Р<001). В парних сравнениях было определено значительное снижение 1-2 группы по сравнению с группами 3-4 с точки зрения неоваскуляризации, плотности фибробластов, отложения коллагена и воспалительных клеток.

\section{Результаты и обсужление}

Механизмы индукции адгезии еще до конца не изучены. Одним из потенциальных триггеров адгезии являются инородные тела, например сетки. Спайки от сетки до большого сальника возникают довольно часто, но, по-видимому, имеют ограниченное значение. В отличие от этого, спайки кишечника к месту десерозированного участка могут привести к серьезным проблемам, наиболее серьезными из которых являются образование свищей, инфекция и кишечная непроходимость [16, с. 71; 17, с. 277]. В экспериментальных исследованиях деперитонизации участка слепой кишки, спровоцировали образование адгезии после удаления имплантата. Поскольку тонкая кишка и слепая кишка подвижны в брюшной полости, гарантирует, что при использовании полепроленой сетки можно ожидать высокую вероятность с возникновением спаек [6, с. 89].

Таким образом, из 24 экспериментальных собак у 17 $(70,8 \%)$ с непокрытой амнеотической мембраной слепой кишкой, развились 2,3 и 4 класса спайки слепой кишки к месту десерозированного участка. Однако у собак, у которых в проекции слепой кишки имплантата, отмечалась низкая частота спаек 1-2 класса по Nair. При гистологическом исследовании выявило моноклеточный 
мезотелиальный слой, покрывающий имплантат, что свидетельствует о висцеральном ре-перитонеализации, как признак заживления брюшины в течение двух недель. Интраабдоминальные сальником спайки особенно часто возникали в области послеоперационного рубца. Мы считаем, что эти спайки сопоставимы с сальниковыми спайками у пациентов [13, с. 880].

Послеоперационные спайки сальника к месту десерозированному участку наблюдали у $(79,1 \%)$ и лапаратомному рубцу наблюдали у (91,6\%). Гистологические спайки характеризовались преобладанием плотности $9,8 \pm 6,9 \%(0,7 \pm 0,08$ мкм3) соединительной ткани, отсуствием на их поверхности мезителиального покрова (12,5\%), выраженным неоангиогенезом (33,3\%), основную часть представляли артериоли $(70,5 \%)$ у капилляры (33,3\%).

Это исследование вводит модель, имитирующую клиническую ситуацию, когда большие десерозированные участки вступает в контакт с нормальными и де-перитонеализованными висцеральными органами [20, с. 334; 22 , с. 284]. Однако тяжелые спайки развиваются с высокой воспроизводимостью, когда отсутствует перитонеальное покрытие. Это свидетельствует о том, что целостность брюшины является решающим фактором для формирования адгезии кишечника к месту десерозированного участка [22, с. 285]. Мы предлагаем нашу модель в качестве подходящей основы для тестирования модели адгезиогенеза брюшной полости.

\section{Выво $\triangle$}

В настоящем исследовании представлена модель, имитирующая клиническую ситуацию моделирование спаечной кишечной непроходимости с применением полипроленовой сетки. Была оценена в эксперименте рол адгезиогенезе. Наблюдалась значительная разница в средних показателях адгезии между группами амнеотической мембраной, 5-фторурацилом $(3,5+0,17)$ и $(5,89$ $\pm 0,91)$ соответственно ( $p=0,0001)$ и между мезогелем и $5 \%$ глюкозой $(9,97 \pm 1,05)$ и $(8,91 \pm 0,99)$ соответственно $(\mathrm{p}=0,001)$. В соотношения фиброза 1 и 2 группы ( $\mathrm{p}=$ $0,002)$ и воспаления $(p=0,003)$ и 3-4 группы $(p=0,003$ и $\mathrm{p}=0,002$ ) соответственно. Таким образом, в результате проведенного исследования можно прийти к следующим выводам:

1. Вэкспериментальной модели, применениедля перетонизации десерозированных участков кишка амниотической мембраны у животных показало более высокую его эффективность по сравнению средствами противоспаечного барьера.

2. Экспериментальные исследования показывает, что процесс адгезиолизиса является обратимым, что вселяет надежду о избирательном развитии процесса и возможность решения процесса поиском эффективных средств против адгезиолизиса.

3. Репаративный процесс дефекта стенки кишки, покрытый имплантантом, протекает минимально выраженными воспалительными реакциями.

\section{ЛИТЕРАТУРА}

1. Ашурметов Р.И., Ризаходжаев А. А. Моделирование спаечной болезни // Вопросы медицины: теория и практика: материалы международной заочной научно-практической конференции. (03 декабря 2012 г.). Новосибирск: СибАК, 2012.

2. Бондаревский И.Я., Шамагамбетов М. С., Бордуновский В. Н. Оценка эффективности средств профилактики после операционного адгезиогенеза брюшин в эксперименте // Клиническая и экспериментальная хирургия им. академ. Б. В. Петровского. 2017. № 2.

3. Дубовиков А.С., Гаврилюк И. О., Куликов А. Н. 0 применении культивированных на амниотической мембране стволовых клеток роговичного эпителия эксперимент // Практическая медицина. 2017. Том 2. № 9.

4. Родин 0.В., Колосунин И. А., Козлов С. А., Паркин П. Н., Мильшева А. Н. Применения амниотической мембраны в лечении диабетических язв стопы (обзор литературы) // Естественные и технические науки. 2020. № 5.

5. Сопуев А.А., Маматов Н. Н., Овчаренко К. Е. Оценка эффективности различных вариантов моделирования спаечного процесса брюшной полости // Актуальные проблемы гуманитарных и естественных наук. 2011. № 3.

6. Altinli E., Sumer A., Koksal N. Prevention of adhesion to prosthetic mesh: comparison of oxidized generated cellulose, Crpolyethylene glycol and hylan G-F 20 // Ulusal Travma Ve Acil Cerrahi Dergisi. 2011. No. 17.

7. Bageacu S., Blanc P., Breton C. Laparoscopic repair of incisional hernia: a retrospective study of 159 patients // Surgical Endoscopy. 2002. No. 16.

8. Dinsmore R.C., Calton W. C. Jr, Harvey S. B., Blaney M.W. Prevention of adhesions to polypropylene mesh in a traumatized bowel model // Journal of the American College of Surgeons. 2000. Vol. 191.

9. Felemovicius I., Bonsack M. E., Hagerman G., Delaney J.P. Prevention of adhesions to polypropylene mesh // Journal of the American College of Surgeons. 2004 Vol. 198.

10. Franconi F., Roux J., Lefebvre-Lacoeuille C., Lemaire L. Imaging visceral adhesion to polymeric mesh using pneumoperitoneal-MRI in $\mathrm{Cr}^{-}{ }^{\prime} \mathrm{j}$ an experimental rat model // Surgical Endoscopy. 2015. Vol. 29.

11. Gaertner W.B., Bonsack M. E., Delaney J. P. Visceral adhesions to hemia prostheses // Hemia. 2010. Vol. 14.

12. Hoffmann N.E., Siddiqui S. A., Agarwal S. Choice of hemostatic agent influences adhesion formation in a rat cecal adhesion model // The Journal of Surgical Research. 2009. №. 155. 
13. Kraemer B., Wallwiener C., Rajab T. K. Standardised models for inducing experimental peritoneal adhesions in female rats. // BioMed Research International. 2014. Vol. 2014. Article number 435056.

14. Lauder C.I., Garcea G., Strickland A., Maddem G. J. Use of a modified chitosan-dextran gel to prevent peritoneal adhesions in a rat model // The Journal of Surgical Research. 2011. No. 171.

15. Matoba M., Hashimoto A., Tanzawa A. Prevention of polyglycolic acid-induced peritoneal adhesions using alginate in a rat model // BioMed Research International. 2015. Vol. 2015. Article number 403413.

16. Parker M.C., Wilson M. S., van Goor H. Adhesions and colorectal surgery-call for action // Colorectal Disease. 2007. Vol. 9(Suppl 2).

17. Peeters E., van Bameveld K.W., Schreinemacher M. H. One-year outcome of biological and synthetic bioabsorbable meshes for augmentation of large abdominal wall defects in a rabbit model // Journal of Surgical Research. 2013. Vol. 180.

18. Poehnert D., Abbas M., Kreipe H. High reproducibility of adhesion formation in rat with meso-stitch approximation of injured cecum and abdominal wall // International Journal of Medical Sciences. 2015 №. 12.

19. Poehnert D., Abbas M., Kreipe H. H., Klempnauer J., Winny M. High reproducibility of adhesion formation in rat with meso-stitch approximation of injured cecum and abdominal wall // International Journal of Medical Sciences. 2015. Vol. 12.

20. Stommel M.W., Strik C., van Goor H. Response to pathological processes in the peritoneal cavity-sepsis, tumours, adhesions, and ascites // Seminars in Pediatric Surgery. 2014. Vol. 23.

21. Wallwiener M., Brucker S., Hierlemann H. Innovative barriers for peritoneal adhesion prevention: liquid or solid? Aratuterine horn model // Fertility and Sterility. 2006. Vol. 86. Sup. 4.

22. Zinther N.B., Wara P., Friis-Andersen H. Intraperitoneal onlay mesh: an experimental study of adhesion formation in a sheep model // Hemia. 2010. Vol. 14.

(c) Магомедов Мухума Магомедович ( muxuma@mail.ru ),

Магомедов Магомед Арсеневич ( magomedovma@rushydro.ru ), Магомедов Магомед Абдулхаликович ( kafedra.khirurgii@mail.ru ).

Журнал «Современная наука: актуальные проблемы теории и практики»

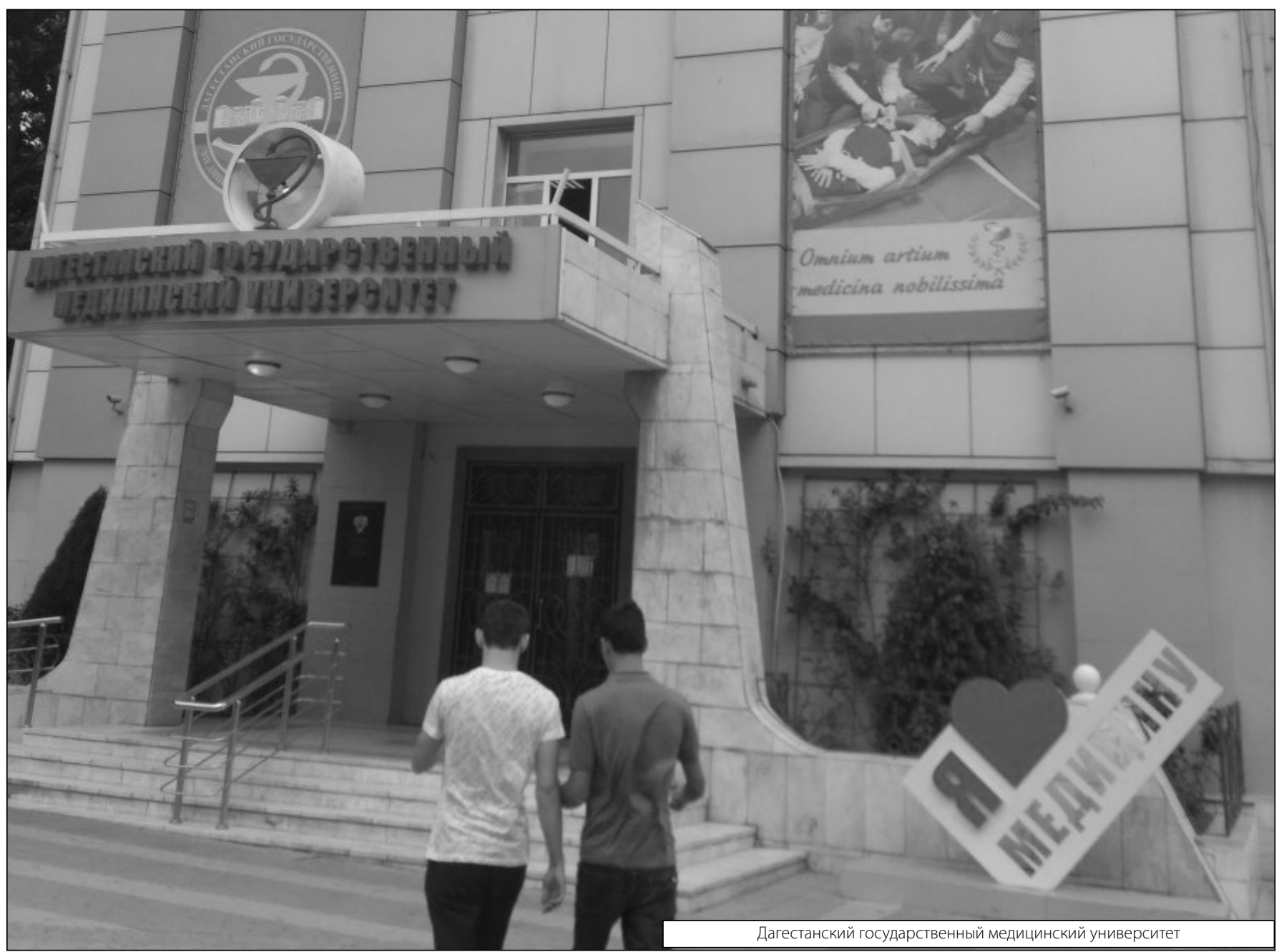

